submitted to Analytical Chemistry on March 12, 2007 (revised on May 31, 2007)

\title{
Retention Mechanism in Reversed-Phase Liquid Chromatography: A Molecular Perspective
}

\author{
Jake L. Rafferty ${ }^{1}$, Ling Zhang ${ }^{1}$, J. Ilja Siepmann ${ }^{1, *}$, Mark R. Schure ${ }^{2}$ \\ ${ }^{1}$ Departments of Chemistry and of Chemical Engineering and Material Science, \\ University of Minnesota, 207 Pleasant Street SE, Minneapolis, MN 55455-0431, USA \\ ${ }^{2}$ Theoretical Separation Science Laboratory, Rohm and Haas Company, \\ 727 Norristown Road, P.O. Box 0904, Spring House, PA 19477-0904, USA
}

\section{Supplementary Information}

The supplementary material provides details on the procedures used to compute (i) the number density and distribution coefficient profiles, (ii) the orientational distribution of solute molecules, (iii) the solvent accessible surface area, and (iv) the incremental free energy of transfer.

\footnotetext{
*To whom correspondence should be addressed. E-mail: siepmann@chem.umn.edu
} 


\section{Number density and distribution coefficient profiles}

The number density and distribution coefficient profiles were computed using a bin width in the $z$-direction of $0.45 \AA$ and the data are averaged over the two surfaces in the stationary phase box. $|z|=0$ is the position of the outermost Si atom of the silica substrate. For solvent and solute molecules the profiles were computed based on center of mass positions, while for the $\mathrm{C}_{18}$ chains the profiles were computed as the sum of the individual $\mathrm{CH}_{\mathrm{x}}$ segment positions. The combined density profiles of water and methanol were used in the fitting of the Gibbs dividing surface for the solvent in each system. The Gibbs dividing surface is defined via an equal area construction as the location (relative the silica substrate) where the surface excess of the solvent number density is equal to zero. $S 1$

\section{Orientational distribution of solute molecules}

To determine how the solutes prefer to align themselves relative to the silica surface, one can use the orientational order parameter, $S,{ }^{S 2}$ calculated as

$$
S=\frac{1}{2 n} \sum_{i=1}^{n}\left\langle 3 \cos ^{2} \theta_{i}-1\right\rangle
$$

where $\theta_{i}$ is the angle between the $i^{\text {th }} 1-3$ vector of the solute molecule and the surface normal and $n$ is the number of such vectors in the molecule. This order parameter approaches unity for vectors preferentially aligned perpendicular to the substrate surface, -0.5 for vectors lying parallel to the surface, and zero for a uniform orientation.

The directional preference of the 1-propanol orientation was evaluated via the quantity $\cos \theta_{\mathrm{OH}}$, where $\theta_{\mathrm{OH}}$ is the angle between the $\mathrm{O}-\mathrm{H}$ bond vector and the surface normal. This

quantity will approach -1 if the bond vector prefers to point towards the silica substrate, +1 if the vector points away from the substrate, and zero for uniform orientation. 


\section{Solvent accessible surface area}

The ensemble averaged solvent accessible surface area was computed by dividing the $x-y$ plane of the stationary phase box into a grid. A probe sphere was then placed on each grid point with a $|z|$ position outside of the stationary phase. The probe was moved towards the stationary phase until it made contact with any of the stationary phase $\mathrm{CH}_{x}$ segments. The $|z|$ position of the sphere at the point of this contact was recorded. The series of points generated by this procedure provides a surface whose area can be computed analytically.

A probe sphere diameter of $4.0 \AA$ was chosen since this is roughly the position of first peak in the solvent oxygen-alkysilane carbon and solvent carbon-alkysilane carbon radial distribution functions. The probe sphere was moved towards the stationary phase in increments $0.05 \AA$ and the grid spacing in the $x$ and $y$ directions was $0.05 \AA$.

\section{Incremental free energy of transfer}

The $z$-dependent incremental free energies for the methylene group were computed from the slope of a linear fit to the plot of free energy of transfer versus number of carbons. This fit was carried out on each bin in the free energy profiles for ethane, propane, and $n$-butane. Methane was not included in the fit since it does follow the linear trend of the

other alkanes. The free energy for the hydroxyl group was computed from the average difference in free energy profiles between each primary alcohol and its corresponding $n$ alkane. Free energy profiles for the individual alkanes and alcohols were computed from the logarithm of distribution coefficient profiles. Uncertainties were estimated from the error of the mean of four independent simulations. Incremental free energies of transfer for the bulk $n$-hexadecane/(water-methanol) system were calculated in a similar manner, but using the overall distribution coefficient for transfer from solvent to $n$-hexadecane since, in this system, the quantity is not $z$-dependent. 
Experimental values of the incremental free energies for a similar RPLC system were computed from the data of Barman. ${ }^{S 3}$ This work reports retention volumes for a variety of solutes including a homologous series of alkylbenzenes from toluene to $n$-octylbenzene, $n$-heptane, and 1-heptanol. The study was conducted using a $\mathrm{C}_{18}$ stationary phase with a grafting density similar to the one used in the current study, unprotected silanol groups, a wide range of water-methanol compositions for the mobile phase, and temperatures in the range from 298 to $328 \mathrm{~K}$. The methylene increment was computed from a linear regression on the logarithm of the retention volume versus number of methylene groups and the free energy of the hydroxyl group was computed from the difference between the logarithms of the retention volumes for 1-heptanol and $n$-heptane. Where experiments were not carried out at the exact temperature studied here, the free energy at the desired temperature was extrapolated from a van’t Hoff plot, all of which showed very good linear fits.

It should be pointed out that the retention volume is related to the free energy of retention by

$$
\begin{aligned}
\Delta G & =-R T \ln K \\
& =-R T \ln k^{\prime}-R T \ln \phi \\
& =-R T \ln V_{\mathrm{ret}}^{\prime}-R T \ln \frac{V_{\mathrm{stat}}}{V_{\mathrm{mob}} \cdot V_{\mathrm{dead}}}
\end{aligned}
$$

where $K, k^{\prime}$, and $\phi$ are the distribution coefficient, the net retention factor, and the phase ratio, respectively, for the transfer from the mobile to the stationary phase. $V_{\text {ret }}^{\prime}, V_{\text {stat }}$, $V_{\text {mob }}$, and $V_{\text {dead }}$ are the corrected retention, stationary phase, mobile phase, and dead volume, respectively. When taking the difference between two free energies for the solutes $A$ and $B$, the $V_{\text {stat }}, V_{\text {mob }}$, and $V_{\text {dead }}$ terms cancel out. This leaves

$$
\Delta G_{A}-\Delta G_{B}=-R T \ln \frac{V_{\mathrm{ret}, A}^{\prime}}{V_{\mathrm{ret}, B}^{\prime}} .
$$

Thus, the incremental free energies are independent of the phase ratio or column dead volume and can be compared directly between two different chromatographic systems. 


\section{References}

[S1] A. Zangwill, Physics at Surfaces (Cambridge University Press: Cambridge, 1988).

[S2] P. G. de Gennes, J. Prost, The Physics of Liquid Crystals, 2nd ed. (Clarendon Press, Oxford, 1993).

[S3] B. N. Barman, A Thermodynamics Investigation of Retention and Selectivity in Reversed-Phase Liquid Chromatogrphy, Ph.D., Georgetown University (1986). 